\section{Comunicación, participación y liderazgo en la percepción del clima emocional en un hospital universitario de Andalucía, España}

\author{
Communication, participation and leadership in the \\ perception of the emotional climate in a university \\ hospital in Andalusia, Spain
}

\author{
Comunicação, participação e liderança na \\ percepção do clima emocional em um hospital \\ universitário da Andaluzia, Espanha
}

Alina Danet 1,2

Joan Carles March 1,2

Inmaculada García Romera ${ }^{1}$

\begin{abstract}
${ }^{1}$ Escuela Andaluza de Salud Pública, Granada, España. 2 Ciber Epidemiología y Salud Pública, Granada, España.

Correspondencia

A. Danet

Escuela Andaluza de Salud Pública.

Cuesta del Observatorio 4 Granada/Granada-18011, España.

alina.danet.easp@juntadean dalucia.es
\end{abstract}

\begin{abstract}
The aim of this study was to identify and analyze perceptions and opinions among health professionals in a university hospital in Andalusia, Spain, regarding the emotional climate, leadership style, quality of information, and internal communication. The study also aimed to collect health professionals' suggestions for improving these workplace characteristics. The study included 730 participants and used a quantitative and qualitative methodology. The results reflect a medium-to-low level of emotional climate, correlated with the leadership style and information and internal communication. Statistically significant differences appeared when comparing professional categories and hospital units. The health professionals provided a positive assessment of the administrators' work, although requiring more task-oriented, participative, and affiliative leadership skills.
\end{abstract}

Organizational Culture; Health Management; Health Personnel; Leadership

\section{Resumen}

El objetivo de este artículo es conocer y analizar la valoración del personal de un hospital universitario de Andalucía, España, sobre el clima emocional, el tipo de liderazgo ejercido y la calidad de la información y comunicación interna, así como recoger sus propuestas de mejora. El estudio realizado con 730 profesionales facultativos, enfermeros y auxiliares, usa metodología cuantitativa y cualitativa. Los resultados reflejan la existencia de un clima emocional medio-bajo, que se correlaciona con el estilo de liderazgo y la percepción sobre el grado de información y comunicación. Se observan diferencias estadísticamente significativas en los resultados, en función de las categorías profesionales, así como de la unidad o servicio hospitalario. Las demandas de los y las profesionales reflejan una valoración positiva de sus responsables, aunque se evidencia la necesidad de más habilidades propias de un estilo de liderazgo orientador, participativo y afiliativo.

Cultura Organizacional; Gestión en Salud; Personal de Salud; Liderazgo 


\section{Introducción}

El clima organizativo en el ámbito sanitario es un concepto amplio y complejo, al cual se le asociaron diversas definiciones ${ }^{1}$ y que constituye un tema central en los estudios multidisciplinarios sobre satisfacción laboral 2, calidad de la vida profesional, condiciones de trabajo o estrés laboral 3 . En el modelo contrastado de los cuatro círculos de McClelland 4, junto con el perfil de motivaciones básicas y los requerimientos del puesto, el clima organizativo, condicionado en un $70 \%$ por el tipo de liderazgo, explica entre un 30 y un $40 \%$ de los resultados del equipo de trabajo.

Los valores y normas que, tanto a nivel individual como relacional, marcan la organización del trabajo en equipo se pueden agrupar, según Borrell Carrió 5,6 en tres núcleos: relaciones interprofesionales, existencia de proyecto común y reconocimiento del saber. Según este autor, las tres dimensiones configuran el clima emocional de los equipos de trabajo, concepto que se podría integrar dentro de los estudios sobre clima organizativo, pero centrando la atención en los aspectos relacionales, comunicativos y emocionales de la experiencia laboral.

La bibliografía disponible sobre la percepción del personal sanitario sobre el clima emocional, refleja la prioridad de este concepto para los profesionales de la salud (para facilitar la lectura del texto, se utilizará el genérico “los" para hacer referencia a ambos sexos), así como la interrelación de este factor con la comunicación interna y los resultados de trabajo 7. Asimismo, el estilo de liderazgo, categorizado según Goleman 8 en seis tipos ideales -coercitivo, orientador, afiliativo, participativo, imitativo o capacitador-, se confirma por parte del personal sanitario, como uno de los condicionantes fundamentales de la calidad del clima emocional 9 .

La confluencia de los diversos condicionantes del clima emocional reclama, por tanto, en el contexto institucional y profesional, una coordinación óptima entre la adecuación del tipo de liderazgo y la gestión de los aspectos relacionales, comunicativos y emocionales de la cultura organizativa 10.

En el contexto hospitalario, las dinámicas relacionales, la organización del trabajo, así como la definición y consecución de los objetivos, están sujetos a un alto grado de complejidad y movilizan a categorías profesionales diversas 11 , lo que implica una mayor exposición al desgaste profesional y demanda una gestión organizativa, basada en combinar con éxito comunicación interna, motivación del equipo, adecuación de objetivos, planificación y seguridad en el tra- bajo 12 y un clima emocional óptimo 13. Estudios recientes evidencian que las necesidades y expectativas del personal hospitalario sobre las relaciones intreprofesionales, el tipo de liderazgo o los temas y canales de comunicación, son heterogéneas, registrando diferencias por sexo y categoría profesional 14 . Asimismo, la alta variabilidad de las unidades hospitalarias, con diferencias significativas en la labor asistencial, tipo de paciente atendido y dinámica del trabajo, imprimen vivencias y necesidades diferentes, no sólo en función de las categorías profesionales, sino también de la pertenencia a distintas unidades o servicios hospitalarios 15 . Por otra parte, las características laborales relacionadas con la disponibilidad de recursos, infraestructuras o turnicidad, son aspectos que imprimen mayor homogeneidad en la percepción de las personas que trabajan en los hospitales e inciden de forma más uniforme en la satisfacción laboral de estos trabajadores 16 .

Por tanto, partiendo de que los factores condicionantes del clima emocional en la atención hospitalaria describen una interconexión inseparable y, además, revierten directamente en la seguridad y satisfacción del paciente 17 , conocer y gestionar correctamente estos factores y las dinámicas internas que se establecen entre ellos, puede contribuir al diseño de mejoras en los resultados de trabajo, maximizando así la calidad asistencial.

En este sentido, el presente trabajo tiene el objetivo de conocer y analizar la valoración del personal hospitalario sobre el clima emocional, el tipo de liderazgo ejercido y la calidad de la información y comunicación interna, así como recoger las propuestas de mejora expresadas por los profesionales sanitarios del centro.

\section{Metodología}

Estudio transversal con metodología cuantitativa y cualitativa, usando un cuestionario de autocumplimentación on line.

\section{Participantes}

La población de estudio la constituyó el total del personal asistencial del hospital de primer nivel de Andalucía, España. Criterios de inclusión: ser personal facultativo y de enfermería. Criterio de exclusión: categorías profesionales no asistenciales y personal directivo. El contacto con los participantes se realizó a través de la gerencia del hospital, que informó sobre los objetivos y metodología del estudio, en una reunión con el personal responsable de las unidades y servicios 
asistenciales, a la que también asistió el coordinador del equipo de investigación. A su vez, los y las responsables trasladaron la información sobre el estudio a los miembros de sus equipos, bien a través de reuniones, como por correo electrónico, asegurando la confidencialidad de los datos y proporcionando el enlace al cuestionario en línea, así como ejemplares del cuestionario en papel. La participación en el estudio fue voluntaria. Los profesionales del hospital dispusieron de un mes para contestar a la encuesta, aunque los formularios recibidos una vez transcurrido este plazo, también fueron incluidos en la población del estudio.

\section{Recogida de datos}

El instrumento de recogida de datos fue un cuestionario de elaboración propia, previamente validado y organizado en tres bloques: (1) clima emocional (10 preguntas en escala Likert, de -5 a +5 puntos: 3 preguntas sobre relaciones interprofesionales, 3 preguntas sobre reconocimiento del saber y 4 preguntas sobre el proyecto común), (2) valoración del/de la responsable (5 preguntas en escala Likert, de $-5 \mathrm{a}+5$ puntos, midiendo la capacidad del líder para apoyar, comunicar y facilitar la participación dentro del equipo) y (3) grado de información y comunicación (8 preguntas en escala Likert, de $-5 \mathrm{a}+5$ puntos y 2 preguntas de tipo sí/no, sobre medios y canales de comunicación usados y preferidos). Por último, el cuestionario incluyó 3 preguntas abiertas, solicitando propuestas de mejora para cada uno de los bloques evaluados y un apartado de datos sociodemográfico con las siguientes variables: sexo, edad, antigüedad, categoría profesional y unidad/servicio al que se pertenece.

\section{Análisis}

El análisis cuantitativo de los datos se realizó con el software SPSS 15.0 (SPSS Inc., Chicago, Estados Unidos) e incluyó: (1) descripción de la población participante; (2) análisis descriptivo (media, moda, mediana, mínimo, máximo) de los índices de "clima emocional", "valoración del/de la responsable" e "información/comunicación", calculados como sumatorio de las preguntas de cada bloque y trasladados a escala $-50,+50$ puntos (siendo -50 el valor mínimo para cada índice y +50 el valor máximo); (3) análisis bivariante de los 3 índices, en función de los datos sociodemográficos (comparación de medias y estudios de correlación); (4) estudio de correlación entre los índices calculados; (5) análisis multivariante del índice de clima emocional, a través de un modelo de regresión lineal.
El modelo de regresión tomó como variable independiente el clima emocional y como variables explicativas: sexo, edad, antigüedad, categoría profesional agrupada y unidad/servicio agrupada. La variable cuantitativa "antigüedad" no mostró una tendencia lineal con el "clima emocional” y, como variable categórica no influyó en el conjunto de los coeficientes, siendo irrelevante y no confusora. La variable edad se introdujo en el modelo como variable lineal. Para las variables categóricas "categoría profesional” y "unidad/ servicio", se construyeron variables dummy para cada una de las categorías de respuesta. Las categorías profesionales se agruparon en: "personal facultativo", "personal de enfermería” y "personal auxiliar de enfermería", coincidiendo con los criterios de inclusión. La categorización de la unidades/servicios, generó tres grupos: "unidades asistenciales", "unidades asistenciales de apoyo" y "unidades de apoyo al diagnóstico", conforme con el tipo de actividad asistencial desarrollada. Para el modelo de regresión multivariante final se comprobaron la normalidad y homocedasticidad de los residuos leverage, a través de un histograma de residuos y un gráfico de dispersión con residuos y predicciones que mostraron linealidad y homogeneidad.

El análisis cuantitativo partió de un intervalo de confianza del 95\%, lo que se asoció a una significación estadística de $\mathrm{p}<0,5$.

Las propuestas de mejora se organizaron en categorías emergentes, a través de la técnica de análisis de contenido, realizando un árbol de códigos en el software Nudist Vivo 11.0 (QSR International Pty Ltd., Doncaster, Australia).

\section{Resultados}

\section{Descripción de la población participante}

La población participante abarcó un total de 730 profesionales, cuya distribución por sexo, categoría profesional y unidad o servicio se presenta en la Tabla 1, de elaboración propia. La media de edad es de 47 años (DT 8,9) y la antigüedad media es de 12 años (DT 15,1). El total de las unidades y servicios participantes se listan en la Tabla 2, donde también se incluye el porcentaje de participación en el estudio, en función de cada unidad/servicio, calculado con la fórmula: número total de participantes en el estudio de cada unidad/servicio, dividido entre el número total de profesionales de cada uni$\mathrm{dad} /$ servicio $^{*} 100$. 
Tabla 1

Distribución de la población participante por sexo, categoría profesional y unidad/servicio.

\begin{tabular}{lccc}
\hline Variable/Categorías & $\mathbf{n}$ & $\%$ & $\%$ válido \\
\hline Sexo & & & \\
Mujer & 472 & 64,7 & 68,9 \\
Hombre & 213 & 29,2 & 31,1 \\
NS/NC & 45 & 6,2 & - \\
Total & 685 & 100,0 & 100,0 \\
Categoría profesional & & & - \\
Facultativo/a & 216 & 29,6 & - \\
Enfermería & 300 & 41,1 & - \\
Auxiliar de enfermería & 214 & 29,3 & - \\
NS/NC & - & - & \\
Total & 730 & 100,0 & 86,8 \\
Unidad/Servicio & & & 7,8 \\
Unidades asistenciales & 616 & 84,5 & 5,4 \\
Unidades asistenciales de apoyo & 55 & 7,5 & 100,0 \\
Apoyo al diagnóstico & 38 & 5,2 & - \\
NS/NC & 21 & 2,8 & 100,0 \\
Total & 730 & & \\
\hline
\end{tabular}

NS/NC: no sabe/no contesta.

Tabla 2

Listado completo de unidades y servicios participantes.

\begin{tabular}{lccc}
\hline Tipo de unidad/servicio & $\mathbf{n}$ & $\begin{array}{c}\mathbf{n} \text { total } \\
\text { (unidad/servicio) }\end{array}$ & \% participación \\
\hline Unidades asistenciales & & & \\
Aparato digestivo & 17 & 101 & 16,8 \\
Cirugía plástica y reparadora & 7 & 73 & 9,5 \\
Cardiología & 56 & 78 & 71,7 \\
Cirugía ortopédica y traumatología & 20 & 230 & 8,6 \\
Cirugía ortopédica y traumatología & 20 & 230 & 8,6 \\
Cuidados críticos y urgencias & 168 & 625 & 26,8 \\
Enfermedades infecciosas & 17 & 36 & 47,2 \\
Ginecología/Obstetricia/Partos & 14 & 332 & 4,2 \\
Medicina física y rehabilitación & 19 & 140 & 13,5 \\
Medicina interna & 5 & 119 & 4,2 \\
Medicina preventiva y salud pública & 27 & 65 & 41,5 \\
Médico quirúrgica y de la infancia & 32 & 371 & 8,6 \\
Nefrología & 28 & 121 & 23,1 \\
Neumología & 10 & 65 & 15,4 \\
Neurociencias & 27 & 169 & 15,9 \\
Neurofisiología clínica & 4 & 22 & 18,2 \\
Endocrinología y nutrición clínica & 3 & 30 & 1,0 \\
Oftalmología & 7 & 78 & 8,9 \\
Oncología médica y radioterápica & 27 & 84 & 32,1 \\
\hline (con & & &
\end{tabular}

(continua) 


\begin{tabular}{lccc} 
Tabla 2 (continuación) & n & $\begin{array}{c}\mathbf{n} \text { total } \\
\text { (unidad/servicio) }\end{array}$ & \% participación \\
\hline Tipo de unidad/servicio & & & \\
\hline Unidades asistenciales & 15 & 45 & 33,3 \\
Oral y maxilofacial & 14 & 27 & 51,8 \\
Otorrinolaringología & 7 & 7 & 100,0 \\
Reumatología & 19 & 139 & 13,7 \\
Salud mental & 10 & 69 & 14,5 \\
Urología & & & 40,7 \\
Unidades asistenciales de apoyo & 82 & 201 & 19,2 \\
$\quad$ Bloque quirúrgico & & 26 & 12,7 \\
Apoyo al diagnóstico & 5 & 55 & 6,4 \\
Anatomía patológica & 7 & 125 & 4,3 \\
Farmacia hospitalaria & 8 & 114 & 13,3 \\
Hematología & 5 & 45 & 3,9 \\
Laboratorios & 6 & 154 & - \\
Microbiología y parasitología & 6 & - & 18,3 \\
Radiodiagnóstico & 43 & 3.976 & \\
NS/NC & 730 & &
\end{tabular}

NS/NC: no sabe/no contesta.

\section{- Clima emocional}

Los resultados de clima emocional se presentan en la Tabla 3, donde los resultados significativos estadísticamente están resaltados con asterisco. La media total de clima emocional se sitúa por encima del valor 0 , aunque sólo asciende a los 5 puntos. Las relaciones interprofesionales aparecen como la dimensión más deficitaria del clima emocional, aunque la idea de proyecto común y el reconocimiento del saber también se sitúan en valores reducidos. Las diferencias por sexo no son considerables. El análisis bivariante refleja que los hombres manifiestan mejor clima general y más reconocimiento del saber y calidad de las relaciones interprofesionales. Por su parte, las mujeres puntúan mejor la existencia de un proyecto común, aunque sin diferencias estadísticamente significativas. El análisis comparativo por categoría profesional y unidades/servicios agrupados, revela diferencias estadísticamente significativas: el personal facultativo percibe en mayor medida reconocimiento del saber y sitúa el clima general en valores superiores en comparación con el resto de profesionales. Sin embargo, representan la categoría que menos refleja la existencia de un proyecto común. El personal auxiliar de enfermería invierte estos resultados: son los que más viven el proyecto común, aunque son quienes peores resultados registran en el resto de las variables. El personal titulado en- fermero se sitúa en niveles intermedios, destacando, sin embargo, como los grupos con mejor percepción sobre la calidad de las relaciones interprofesionales.

El análisis comparativo en función de las unidades y servicios agrupados muestra pocas diferencias entre las unidades asistenciales y las de apoyo al diagnóstico, que se mantienen en valores medio-bajos. Comparativamente, las unidades asistenciales de apoyo registran niveles más bajos en prácticamente todas las variables, aunque también es importante tener en cuenta que el número de participantes de estas unidades es más reducido.

El modelo de regresión confirma los mejores niveles de clima emocional entre el personal facultativo. El valor del perfil tomado como constante (hombre, médico, con edad media), refleja un clima emocional reducido, inferior a los 10 puntos en la escala $-50,+50$. La variable "sexo", analizada en el modelo multivariante, muestra una contradicción con los resultados del análisis bivariante: las mujeres suman casi medio punto al clima emocional de los hombres, aunque no se observan diferencias estadísticamente significativas. Por categoría profesional, enfermeros y enfermeras restan 2 puntos a la media del personal médico, diferencia que aumenta a casi 5 puntos en el personal auxiliar de enfermería $(\mathrm{p}<0,05)$. No se perciben diferencias por edad. 
Tabla 3

Resultados de clima emocional.

\begin{tabular}{|c|c|c|c|c|c|}
\hline Variables & Categoría & $\begin{array}{l}\text { Clima emocional } \\
\qquad(-50,+50)\end{array}$ & $\begin{array}{c}\text { Relaciones } \\
\text { interprofesionales } \\
(-15,+15)\end{array}$ & $\begin{array}{l}\text { Reconocimiento } \\
\text { del saber } \\
(-15,+15)\end{array}$ & $\begin{array}{l}\text { Proyecto común } \\
\qquad(-20,+20)\end{array}$ \\
\hline \multirow[t]{3}{*}{ Valores totales } & Total & 5,94 & 0,37 & 3,52 & 2,04 \\
\hline & DT & 10,20 & 4,45 & 6,43 & 5,21 \\
\hline & $\mathrm{N}$ & 715 & 726 & 723 & 720 \\
\hline \multirow[t]{6}{*}{ Sexo } & Mujer & 5,67 & 0,34 & 3,21 * & 2,14 \\
\hline & DT & 10,11 & 4,57 & 6,23 & 5,29 \\
\hline & $\mathrm{N}$ & 461 & 468 & 467 & 464 \\
\hline & Hombre & 6,52 & 0,48 & 4,26 * & 1,78 \\
\hline & DT & 10,49 & 4,44 & 6,54 & 5,17 \\
\hline & $\mathrm{N}$ & 210 & 213 & 212 & 211 \\
\hline Categoría & Facultativo/a & 7,84 * & 0,45 & 5,85 * & 1,52 \\
\hline \multirow[t]{9}{*}{ profesional * } & DT & 8,16 & 4,16 & 6,07 & 4,46 \\
\hline & $N$ & 211 & 215 & 213 & 214 \\
\hline & Enfermería & 5,95 * & 0,56 & 3,18 * & 2,21 \\
\hline & DT & 10,73 & 4,52 & 6,21 & 5,42 \\
\hline & $\mathrm{N}$ & 297 & 300 & 299 & 298 \\
\hline & Auxiliar de & 4,00 * & 0,02 & 1,68 * & 2,35 \\
\hline & enfermería & 10,95 & 4,64 & 6,40 & 5,60 \\
\hline & DT & 207 & 211 & 211 & 208 \\
\hline & $\mathrm{N}$ & & & & \\
\hline \multirow[t]{11}{*}{ Unidad/Servicio * } & Unidades & 5,89 & 0,32 & 3,46 & 2,12 \\
\hline & asistenciales & 10,10 & 4,53 & 6,29 & 5,13 \\
\hline & DT & 602 & 612 & 610 & 606 \\
\hline & $\mathrm{N}$ & & & & \\
\hline & Unidades & 4,81 & 0,61 & 2,87 & 1,32 \\
\hline & asistenciales de & 11,24 & 4,38 & 7,01 & 5,49 \\
\hline & $\begin{array}{l}\text { apoyo } \\
\text { DT } \\
\text { N }\end{array}$ & 55 & 55 & 55 & 55 \\
\hline & Apoyo al & 6,91 & 0,44 & 4,16 & 2,21 \\
\hline & diagnóstico & 10,21 & 3,65 & 7,69 & 5,20 \\
\hline & DT & 37 & 38 & 37 & 38 \\
\hline & $\mathrm{N}$ & & & & \\
\hline \multirow[t]{6}{*}{ Modelo de regres } & & & & Coeficiente B & $\begin{array}{l}\text { Significación } \\
\text { estadística }\end{array}$ \\
\hline & Constante: perso & nal médico, hombre, & unidad asistencial & 6,32 & - \\
\hline & & Sexo & & 0,454 & 0,64 \\
\hline & & Enfermería & & $-2,02$ * & 0,05 \\
\hline & & Auxiliares enfermería & & $-4,73 *$ & 0,00 \\
\hline & & Edad & & 0,03 & 0,50 \\
\hline
\end{tabular}

* $p<0,05$ (dato estadísticamente significativo).

\section{- Valoración de responsables}

La evaluación de los líderes, pese a no alcanzar un valor alto, indica un liderazgo positivo. Las diferencias entre las opiniones de hombres y muje- res no son significativas y la categoría profesional no imprime tampoco una valoración diferente sobre el personal responsable. Las unidades incluidas dentro del grupo de apoyo al diagnóstico refieren una valoración levemente superior de 
sus responsables en comparación con el resto de unidades y servicios, que se sitúan en valores medios similares. Además, estos valores diferenciales en función de unidad/servicio, incluidas en la Tabla 4, de elaboración propia, son estadísticamente significativos $(\mathrm{p}<0,05)$.

\section{- Calidad de la información y comunicación}

El grado de información y comunicación ronda el valor medio de 12 puntos. Las mujeres suman casi un punto a la media de los hombres, aunque la diferencia no es estadísticamente significativa. Por categoría profesional, el personal facultativo indica un mayor grado de información y comunicación. Las unidades o servicios que se consideran peor informados son los asistenciales.

El estudio de correlación entre las variables cuantitativas, presentado en la Tabla 5, de ela- boración propia, muestra la existencia de una relación directamente proporcional del clima emocional, especialmente en su subvariable de "reconocimiento del saber", con la valoración del/de la responsable, así como con el grado de “información/comunicación”. Los resultados que presentan significación estadística están marcados con asterisco en la Tabla 5. Por otra parte, la valoración de los responsables es la cuestión que más incide en la percepción sobre la calidad de la información y comunicación (índice de correlación superior a 0,700, lo que representa una muy alta asociación entre variables). El apoyo que los responsables ofrecen a su equipo imprime una mayor cohesión grupal, en torno a un proyecto común. Las oportunidades de participación, favorecidas por el tipo de liderazgo, conllevan una mejora de la información y comunicación del personal sanitario, aunque

Tabla 4

Valoración del/de la responsable e información/comunicación.

\begin{tabular}{|c|c|c|c|}
\hline Variables & Categoría & $\begin{array}{l}\text { Valoración responsable } \\
\qquad(-50,+50)\end{array}$ & $\begin{array}{l}\text { Información/Comunicación } \\
\qquad(-50,+50)\end{array}$ \\
\hline \multirow[t]{3}{*}{ Valores totales } & Total & 12,34 & 12,15 \\
\hline & DT & 10,50 & 11,16 \\
\hline & $\mathrm{N}$ & 709 & 706 \\
\hline \multirow[t]{6}{*}{ Sexo } & Mujer & 12,54 & 12,56 \\
\hline & DT & 10,44 & 11,50 \\
\hline & $\mathrm{N}$ & 461 & 460 \\
\hline & Hombre & 11,64 & 11,91 \\
\hline & DT & 10,83 & 10,54 \\
\hline & $\mathrm{N}$ & 203 & 201 \\
\hline \multirow[t]{9}{*}{ Categoría profesional } & Facultativo/a & 12,47 & 13,15 \\
\hline & DT & 10,67 & 10,07 \\
\hline & $\mathrm{N}$ & 202 & 203 \\
\hline & Enfermería & 12,15 & 11,84 \\
\hline & DT & 10,56 & 10,50 \\
\hline & $\mathrm{N}$ & 297 & 295 \\
\hline & Auxiliar de enfermería & 12,49 & 11,61 \\
\hline & DT & 10,29 & 12,91 \\
\hline & $\mathrm{N}$ & 210 & 208 \\
\hline \multirow[t]{10}{*}{ Unidad/Servicio } & Unidades asistenciales & 12,04 & 11,96 \\
\hline & DT & 10,49 & 11,21 \\
\hline & $\mathrm{N}$ & 597 & 594 \\
\hline & Unidades asistenciales de & 13,55 & 13,15 \\
\hline & ароуо & 9,00 & 9,31 \\
\hline & DT & 54 & 54 \\
\hline & $\mathrm{N}$ & & \\
\hline & Apoyo al diagnóstico & 14,37 & 13,42 \\
\hline & DT & 12,06 & 12,54 \\
\hline & $\mathrm{N}$ & 38 & 38 \\
\hline
\end{tabular}


Análisis de correlación entre variables lineales.

\begin{tabular}{|c|c|c|c|c|c|}
\hline & Información & Clima emocional & $\begin{array}{l}\text { Relaciones } \\
\text { interprofesionales }\end{array}$ & $\begin{array}{l}\text { Reconocimiento } \\
\text { del saber }\end{array}$ & Proyecto común \\
\hline Valoración responsable & 0,732 * & 0,357 * & $-0,111$ * & 0,559 * & 0,100 * \\
\hline Apoya a su equipo & 0,614 * & $0,301 *$ & $-0,100 *$ & 0,084 & 0,614 * \\
\hline Mantiene informado al equipo & 0,675 * & $0,321 *$ & $-0,059$ & 0,466 * & 0,102 * \\
\hline $\begin{array}{l}\text { El personal tiene oportunidades de } \\
\text { participación }\end{array}$ & 0,702 * & 0,379 * & $-0,067$ & 0,558 * & 0,105 * \\
\hline El equipo conoce sus funciones & 0,625 * & 0,288 * & $-0,129 *$ & $0,499 *$ & 0,055 \\
\hline El equipo lo considera un buen líder & 0,553 * & 0,268 * & $-0,114 *$ & 0,420 * & 0,092 \\
\hline Información/Comunicación & - & 0,360 * & $-0,141$ * & 0,549 * & 0,110 * \\
\hline
\end{tabular}

* $p<0,05$ (dato estadísticamente significativo).

inciden de forma indirectamente proporcional en la calidad de las relaciones interprofesionales percibidas.

\section{Propuestas de mejora}

Del total de 730 profesionales que participaron en el estudio, 66 formularon propuestas de mejora, de los cuales 59 especificaron dos propuestas y 7 personas aportaron tres propuestas. A pesar de que las propuestas de mejora que se solicitaban se referían a los tres bloques del cuestionario, el análisis cualitativo reflejó que las demandas y necesidades que el personal participante enunció no respondían a esta clasificación, y podían organizarse en torno a tres categorías emergentes: (1) aspectos relacionados con la especificidad y organización del trabajo (objetivos, agendas, infraestructuras, formación, características de la práctica asistencial); (2) aspectos de interacción horizontal (relación con compañeros, comunicación, participación e identidad profesional); (3) aspectos de interacción vertical (relación con superiores, información y comunicación, motivación, tipo de liderazgo).

\section{- Aspectos relacionados con el trabajo}

La mayoría de las propuestas de este bloque (27 citas) se refieren a la necesidad de aumentar el personal laboral y disminuir las agendas de trabajo, reduciendo así la carga laboral de los trabajadores: "Aumentar la plantilla de manera que la carga de trabajo sea menor y por tanto, el estrés también" (mujer, enfermera, unidad asistencial).

En segundo lugar, los profesionales destacan la necesidad de mayor incentivación económica, ajustada a la definición de objetivos a cumplir (22 citas). "Incremento salarial" (propuesta anónima), “mejorar el salario” (mujer, enfermera, unidad asistencial), son algunas de las fórmulas que reiteran la propuesta de compensación salarial.

La necesidad de acondicionar el sitio de trabajo, las infraestructuras y tecnologías es un aspecto destacado, que los participantes asocian a su salud y seguridad laboral (18 citas). Las principales demandas se refieren al instrumental tecnológico utilizado: "agilizar las reparaciones" (propuesta anónima), dotar de "más equipamiento para el cuidado del paciente" (hombre, enfermero, unidad asistencial). Y en segundo lugar, se reclama una mejor adecuación del espacio laboral, argumentándose que "lo insalubre del espacio físico donde se desarrolla la labor asistencial incide negativamente en el clima emocional del equipo" (mujer, enfermera, unidad asistencial) y que "para un buen clima emocional es imprescindible que la ergonomía y la salud laboral se tengan en cuenta" (hombre, auxiliar de enfermería, unidad asistencial).

La necesidad de adaptar las líneas formativas a las prioridades y demandas de los profesionales se hace patente en 8 citas, que resaltan la demanda de formación de tipo profesional, pero también en aspectos relacionales y emocionales: "formación individualizada ajustándola al puesto de trabajo" (mujer, no sabe/no contesta), "cursos de inteligencia emocional y habilidades emocionales" (mujer, auxiliar de enfermería, unidad asistencial).

Por último, la interacción con los pacientes, específica del trabajo asistencial, se considera como dificultad añadida y carga emocional adicional (5 citas): "No nos olvidemos que trabajamos para personas con sus necesidades y problemas de salud. Debemos ayudarles y hacerles lo menos do- 
loroso posible su paso por el centro" (enfermera, unidad de apoyo al diagnóstico).

\section{- Aspectos de interacción horizontal}

La importancia del compañerismo es identificada como un pilar del buen clima laboral por todos los profesionales. Sólo 6 citas muestran la satisfacción con la calidad de las relaciones entre iguales ("la suerte de este hospital es el personal que tiene, que somos divinos", mujer, auxiliar de enfermería, unidad asistencial), mientras que otras 10 opiniones expresan la necesidad de mejorar la empatía entre los trabajadores ("La mejora sería por parte de los profesionales, que deberíamos saber aprovechar mejor las oportunidades que se nos dan, compartir y no mirarnos todos el ombligo", mujer, enfermera, unidad asistencial).

La participación igualitaria en los equipos de trabajo multiprofesionales es definida como una línea de mejora destacada dentro del hospital. En este sentido, cada categoría profesional manifiesta necesidades específicas en función de su autopercepción dentro del equipo, así como en base a las expectativas de interacción con el resto de categorías. El personal de enfermería y auxiliar son los que principalmente demandan un mejor status y valoración dentro del equipo.

"Dar oportunidades a los enfermeros de a pie, no sólo a los mandos intermedios; todas las opiniones son útiles, sobre todo de las personas que pueden transmitir sus experiencias con los pacientes" (mujer, enfermera, unidad asistencial).

"Las auxiliares de enfermería nos sentimos discriminadas con el resto del hospital" (mujer, auxiliar de enfermería, unidad asistencial).

Las opiniones vertidas en torno a la información transmitida horizontalmente, revelan la disconformidad del personal ante un uso excesivo de los circuitos de comunicación informales: "Evitar la información de pasillo, hay que acabar con los cortijos" (mujer, enfermera, unidad asistencial); "que no te enteres de las cosas de bola en bola, sino crear un circuito correcto" (propuesta anónima).

\section{- Aspectos de interacción vertical}

La relación con el personal responsable es identificada por la mayoría de los profesionales como la cuestión más importante en la gestión de los aspectos relacionales y comunicacionales dentro de los equipos hospitalarios. 54 citas describen la necesidad de maximizar la eficiencia y adecuación de los estilos de liderazgo, tanto en los cargos intermedios, como entre los responsables. Entre las principales competencias de los líderes, los participantes en el estudio destacan: a) Establecer y mantener un grado óptimo de información entre los miembros del equipo, especialmente a través de reuniones con todas las categorías profesionales (20 citas); "Estandarizar con carácter obligatorio reuniones interdisciplinares, donde todo se expresen y no sea posible responsabilizar de los problemas a quienes no estén presentes" (mujer, enfermera, unidad asistencial).

b) Facilitar la bidireccionalidad del flujo comunicacional, transmitiendo y recibiendo información (14 citas); "Más reuniones de trabajo donde se nos informe y podamos también decir lo que pensamos" (mujer, auxiliar de enfermería, apoyo al diagnóstico).

c) Promover la participación de todo el personal, ofrecer un trato equitativo y tener en cuenta las opiniones de los trabajadores (10 citas); “Considero que deberían atenderse los propuestas de mejora que hacemos las profesionales, que actualmente no son escuchadas y que son reflejo de los problemas asistenciales redundando en conflictividad emocional en nuestro lugar de trabajo" (mujer, médico, unidad asistencial).

“Tener en cuenta las opiniones de todos los trabajadores, que se lleven a cabo las propuestas que se hacen en las reuniones con los superiores" (mujer, auxiliar de enfermería, unidad asistencial de apoyo).

d) Motivar e incentivar al personal, mostrar interés y empatía (10 citas). "Desarrollar estrategias que motiven al personal, por ejemplo realizar cursos donde se eleve la autoestima del personal, dotar al trabajador de los suficientes recursos humanos y materiales para que pueda llevar a buen término su trabajo..." (mujer, auxiliar de enfermería, unidad asistencial).

"La dirección debe dar imagen de escucha del personal, entenderlo, mostrar interés, tener humanidad y acercamiento, todos somos humanos" (hombre, enfermero, unidad asistencial).

\section{Discusión}

El estudio confirma el tipo de liderazgo ejercido como uno de los principales factores condicionantes del clima emocional, en los equipos de trabajo de la atención sanitaria especializada. Asimismo, el grado de información y comunicación intrahospitalarias se asocia a las responsabilidades y funciones de los coordinadores de los equipos. De aquí que la valoración de los líderes y de la información y comunicación sean similares (valores medios), mientras que la calidad del clima emocional se considere de forma más crítica, según muestran los resultados cuantitativos. Las opiniones de los pro- 
fesionales participantes, recogidas en la parte cualitativa, apoyan este argumento, al reflejar que la insuficiente disponibilidad de recursos o las infraestructuras deficitarias tienen un peso negativo importante en la percepción del clima emocional a nivel laboral. La importancia de disponer de condiciones laborales adecuadas, objeto de estudio de una investigación realizada recientemente a nivel europeo, pone de manifiesto que una consideración insatisfactoria del contexto en el cual se desarrolla el trabajo asistencial hospitalario, puede generar incluso la intención de abandonar la práctica profesional 18. Por tanto, una primera línea de intervención podría definirse en torno a la adecuación de las condiciones y relaciones profesionales en base a las demandas de los profesionales de los centros asistenciales.

En este sentido, la combinación de metodología cuantitativa y cualitativa ofrece una visión más completa sobre las necesidades de las personas que participan en el estudio, ya que permite conocer la relación entre liderazgo y clima emocional, al mismo tiempo que comprobar, enriquecer y contextualizar los resultados obtenidos 19. Sin embargo, el estudio tiene la limitación de la baja participación de los profesionales, tanto en la cumplimentación del cuestionario en línea, como en la generación de propuestas de mejora. La reducida participación podría ser interpretada como un indicador de la baja receptividad e implicación del personal sanitario, así como de cierto grado de apatía y desinterés general, posiblemente agudizados por las restricciones y recortes realizados en el actual contexto de crisis económica y que han afectado sensiblemente la gestión de la sanidad española 20,21,22. Sin embargo, ante la escasa investigación realizada sobre esta cuestión, se haría necesario indagar con más profundidad en las consecuencias de la crisis económica en la gestión sanitaria.

Retomando la clasificación de los tipos de liderazgo de Goleman 8, la situación de crisis indicaría la necesidad de un liderazgo orientador, que dote de una nueva visión a largo plazo y en el que la dirección ofrezca pautas claras de actuación para el personal. En la línea del liderazgo orientador, podemos situar la necesidad expresada por los participantes en el estudio de percibir una motivación más directa y activa por parte de sus responsables. Sin embargo, las propuestas del personal encuestado visibilizan también las demandas sobre habilidades de liderazgo afiliativo y participativo. Dentro del liderazgo afiliativo se identifica como principal estrategia de comunicación y consenso la reunión multidisciplinar, que los trabajadores consideran clave para mejo- rar las relaciones interprofesionales y armonizar el trabajo en equipo. Por otra parte, a la necesidad de consenso se suma también la apuesta por el proyecto común. Todas las categorías profesionales describen en este sentido la exigencia de un liderazgo que fomente el compromiso, así como la participación de todos y todas en generar ideas e iniciativas útiles para gestionar la actividad en equipo.

Frente a estas demandas que combinan liderazgo orientador, participativo y afiliativo, el estudio refleja también la heterogeneidad en la valoración sobre la calidad del clima emocional, observándose diferencias relevantes entre las unidades y servicios participantes, así como entre categorías profesionales. En los equipos asistenciales se evidencian relaciones interprofesionales satisfactorias, especialmente para la categoría profesional de enfermería, que, según refleja la bibliografía disponible, pese al alto grado de desgaste emocional asociado a la actividad asistencial 15,23,24 que puede generar una sobrecarga del rol profesional 25 , encuentran gratificante el trabajo directo con los pacientes y establecen más vínculos emocionales dentro del equipo 26. Sin embargo, en los equipos asistenciales no existe un proyecto común suficientemente consolidado y el reconocimiento del saber se considera óptimo sólo por parte del personal facultativo, lo que puede representar una limitación para la correcta gestión del equipo 27 .

El hecho de que no todos los perfiles profesionales sientan que su labor es reconocida dentro de los equipos multidisciplinarios, indica la persistencia del modelo jerárquico tradicional dentro de la organización hospitalaria y puede explicar las tensiones interprofesionales 11. El estatus médico privilegiado imprime relaciones interprofesionales desiguales y una estructura de poder hegemónico, donde las contradicciones y conflictos alrededor de las identidades profesionales son comunes y de difícil resolución 28,29. En este sentido, promover un modelo de gestión más igualitario que permita la participación y reconocimiento de todos los agentes implicados, podría ser una alternativa eficaz al modelo tradicional 13, lo que también generaría una mejor satisfacción laboral de todo el personal 30 .

La dificultad de cohesionar el equipo hospitalario, dificultado por la heterogeneidad de demandas y necesidades vertidas por categorías profesionales variadas, a las que se pueden sumar las opiniones y expectativas de los profesionales que no realizan una labor asistencial, es evidenciada también en otros estudios. Éstos reflejan la existencia de situaciones conflictivas en la interacción interprofesional, explicada en gran parte por dificultades de tipo organizativo 
y por la rigidez de las identidades y roles profesionales 11 .

Por tanto, teniendo en cuenta la variabilidad de las demandas y necesidades de las distintas categorías profesionales en el ámbito hospitalario y, al mismo tiempo, las expectativas coincidentes sobre las habilidades de liderazgo, consideramos que sería necesario indagar con mayor profundidad en la relación del tipo de liderazgo, clima organizacional y resultados de trabajo. Una línea de investigación en este sentido podría ser comparar la autopercepción de los responsables con la visión de los profesionales, para encontrar los puntos de consenso y divergencias y optimizar así los estilos de dirección 31 , ante la evidencia de que, en el contexto asistencial hospitalario, una dirección y coordinación óptima maximizarían la satisfacción de los pacientes 32 .

\section{Resumo}

O objetivo deste artigo foi identificar e analisar a percepção e a opinião de profissionais de um hospital universitário na Andaluzia, Espanha, sobre clima emocional, tipo de liderança e qualidade da informação e comunicação interna, assim como identificar propostas de melhoria. O estudo foi realizado com 730 profissionais de saúde e utilizou metodologia quantitativa e qualitativa. Os resultados refletem a existência de um clima emocional médio-baixo, relacionado com o estilo de liderança e com a percepção sobre o grau de informação e comunicação interna. São observadas diferenças estatisticamente significativas nos resultados, em função das categorias profissionais e unidades ou serviço hospitalar. As demandas dos profissionais refletem uma valoração positiva de seus líderes, apesar de evidenciar a necessidade de habilidades próprias de um estilo de liderança orientador, participativo e afiliativo.

Cultura Organizacional; Gestão em Saúde; Pessoal de Saúde; Liderança

\section{Colaboradores}

A. Danet contribuyó con el marco teórico, búsqueda bibliográfica, análisis de datos, redacción del manuscrito y revisión del manuscrito. J. C. March contribuyó con el marco teórico y revisión del manuscrito. I. G. Romera contribuyó con el marco teórico, revisión bibliográfica, redacción del manuscrito y revisión del manuscrito.

\section{Agradecimientos}

Agradecemos a los revisores los comentarios realizados que han contribuido a mejorar el manuscrito. 


\section{Referencias}

1. Delgado Sánchez A, Bellón Saameño JA, MartínezCañavate López-Montes MT, Luna del Castillo JD, López Fernández LA, et al. Las dimensiones del clima organizacional percibidas por los médicos de familia. Aten Primaria 2006; 37:489-97.

2. Robles-García M, Dierssen-Sotos T, MartínezOchoa E, Herrera-Carral P, Díaz-Mendi AR, LlorcaDíaz J. Variables relacionadas con la satisfacción laboral: un estudio transversal a partir del modelo EFQM. Gac. Sanit. 2005; 19:127-34.

3. Grau A, Suñer R, García M. El optimismo de los profesionales sanitarios y su relación con la calidad de vida, el burnout y el clima emocional. Rev Cal Asist 2005; 20:370-6.

4. McClelland DC. A competency model for human resource management specialist to be used in the delivery of the human resource management cycle. Boston: McBer; 1975.

5. Borrell Carrió F. Competencia emocional del médico. FMC Form Med Contin Aten Prim 2007; 14:133-41.

6. Borrell Carrió F. Cómo trabajar en equipo y relacionarse eficazmente con jefes y compañeros. Barcelona: Ediciones Gestión 2000; 1996.

7. March Cerdá JC, Galiana Auchel JM, Danet Danet A; Equipo Climap Sevilla. Clima emocional en los centros de atención primaria de un distrito sanitario urbano de Andalucía. Enfermería Comunitaria: Revista Internacional de Cuidados de Salud Familiar y Comunitaria 2011; 7(2). http://www.index-f. com/comunitaria/v7n2/ec7509r.php.

8. Goleman D. Leadership that gets result. http:// www.haygroup.com/downloads/fi/leadership_ that_gets_results.pdf (accedido el 25/Nov/2013).

9. García Romera I, Danet Danet A, March Cerdá JC Emotional climate in primary health care teams. A qualitative approach. Rev Cal Asist 2010: 25: 200-6.

10. Martin GP, Waring J. Leading from the middle: constrained realities of clinical leadership in healthcare organization. Health (London) 2013; 17:358-74.

11. Maniou M. Labour conflicts between administrative, medical and nursing personnel in the hospital. Health Science Journal 2012; 6:234-45.

12. Gómez Sánchez MC, Álamo Santos MC, Amador Bohórquez M, Ceacero Molina F, Mayor Pascual A, Muñoz González A, et al. Estudio de seguimiento del desgaste profesional en relación con factores organizativos en el personal de enfermería de medicina interna. Med Segur Trab 2009; 55:52-62.

13. Alonso E, Rubio A, March JC, Danet A. Clima emocional y comunicación interna en una unidad de gestión clínica y dos servicios hospitalarios tradicionales. Rev Cal Asist 2011; 26:281-4.
14. Pera G, Serra-Prat M. Prevalencia del síndrome del quemado y estudio de los factores asociados en los trabajadores de un hospital comarcal. Gac Sanit 2002; 16:480-6.

15. Albaladejo R, Villanueva R, Ortega P, Astasio P, Calle ME, Domínguez V. Síndrome de Burnout en el personal de enfermería de un hospital de Madrid. Rev Esp Salud Pública 2004; 78:505-16.

16. Deschamps Perdomo A, Olivares Roman SB, Rosa Zabala KL, Asunsolo del Barco A. Influencia de los turnos de trabajo y las guardias nocturnas en la aparición del síndrome de burnout en médicos y enfermeras. Med Segur Trab 2011; 57:224-41.

17. Nurok M, Evans LA, Lipsitz S, Satwicz P, Kelly A, Frankel A. The relationship of the emotional climate of work and threat to patient outcome in a high-volume thoracic surgery operating room team. BMJ Qual Saf 2011; 20:237-42.

18. Aiken LH, Sermeus W, Van den Heede K, Sloane DM, Busse R, McKee M, et al. Patient safety, satisfaction and quality of hospital care: cross sectional surveys of nurses and patients in 12 countries in Europe and the United States. BMJ 2012; 344.

19. Zabalegui A. Más allá del dualismo cualitativo- cuantitativo. Enferm Clín 2002; 12:74-9.

20. Zabalegui A, Cabrera E. Economic crisis and nursing in Spain. J Nurs Manag 2010; 18:505-8.

21. González B, Barber P. Sostenibilidad y condiciones de empleo en el Sistema Nacional de Salud. Rev Adm Sanit Siglo XXI 2010; 8:89-100.

22. Peiró S, Meneu R. Crisis económica y epicrisis del sistema sanitario. Aten Primaria 2011; 43:115-6.

23. Adali E, Priami M, Evagelou H, Mougia V, Ifanti M, Alevizopoulos G. Síndrome del quemado en el personal de enfermería psiquiátrica de hospitales griegos. Eur J Psychiat 2003; 17:161-70.

24. Navarro Arnedo JM. Revisión de los estudios sobre los profesionales de enfermería de las unidades de cuidados intensivos de España. Enferm Glob 2012; 11:267-89.

25. Moreno B, Peñacoba C. El estrés asistencial en los servicios de salud. In: Simón MA, editor. Manual de psicología de la salud. Fundamentos, metodología y aplicaciones. Madrid: Biblioteca Nueva; 1999. p. 739-64.

26. Sandelans LE, Boudens CJ. Feeling at work. In: Fineman S, editor. Emotions in organizations. London: Sage; 2002. p. 46-63.

27. Richards A, Carley J, Jenkins-Clarke S, Richards DA. Skill mix between nurses and doctors working in primary care- delegation or allocation: a review of the literature. Int J Nurs Stud 2000; 37:185-97. 
28. Martin DR, O'Brien JL, Heyworth JA, Meyer NR. Point counter-point: the function of contradiccions on an interdisciplinary health care team. Qual Health Res 2008; 18:369-79.

29. Elston S, Holloway I. The impact of recent primary care reforms in the UK on interprofessional working in primary care centres. J Interprof Care 2001; 15:19-27.

30. Lu H, Barriball KL, Zhang X, White A. Job satisfaction among hospital nurses revisited: a systematic review. Int J Nurs Stud 2012; 49:1017-38.

31. Vesterinen S, Suhonen M, Isola A, Paasivaara L. Nurse managers' leadership styles in Finland. Nurs Res Pract 2012; 2012:605379.
32. Boev C. The relationship between nurses' perception of work environment and patient satisfaction in adult critical care. J Nurs Scholarsh 2012; 44:368-75.

Recibido el 22/Nov/2013

Versión final presentada el 26/Ago/2013

Aprobado el 04/Oct/2013 